\title{
Strategic Games and Truly Playable Effectivity Functions
}

\author{
Valentin Goranko \\ Informatics and Mathematical \\ Modelling, Technical \\ University of Denmark \\ vfgo@imm.dtu.dk
}

\author{
Wojciech Jamroga \\ Computer Science and \\ Communication, University of \\ Luxembourg \\ wojtek.jamroga@uni.lu
}

\author{
Paolo Turrini \\ Information and Computing \\ Sciences, Utrecht University \\ paolo@cs.uu.nl
}

\begin{abstract}
A well known (and often used) result by Marc Pauly states that for every playable effectivity function $\mathrm{E}$ there exists a strategic game that assigns to coalitions exactly the same power as $\mathrm{E}$, and vice versa. While the latter direction of the correspondence is correct, we show that the former does not always hold in the case of infinite game models. We point out where the proof of correspondence goes wrong, and we present examples of playable effectivity functions in infinite models for which no equivalent strategic game exists. Then, we characterize the class of truly playable effectivity functions, that does correspond to strategic games. Moreover, we discuss a construction that transforms any playable effectivity function into a truly playable one while preserving the power of most (but not all) coalitions. We also show that Coalition Logic is not expressive enough to distinguish between playable and truly playable effectivity functions, and we extend it to a logic that can make this distinction while enjoying finite axiomatization and finite model property.
\end{abstract}

\section{Categories and Subject Descriptors}

I.2.11 [Artificial Intelligence]: Distributed Artificial Intelligence-Multiagent Systems; I.2.4 [Artificial Intelligence]: Knowledge Representation Formalisms and Methods-Modal logic; J.4 [Social and Behavioral Sciences]: Economics

\section{General Terms}

Theory

\section{Keywords}

Strategic reasoning, cooperative games, correspondence

\section{INTRODUCTION}

Several logics for reasoning about coalitional power have been proposed and studied in the last two decades. Eminent examples are: Alternating-time Temporal Logic (ATL) [1], Coalition Logic (CL) [11], and Seeing To It That (STIT) [2], used in computer science and philosophy to reason about properties of multi-agent systems. A crucial feature of these

Cite as: Strategic Games and Truly Playable Effectivity Functions, Goranko, Jamroga, Turrini, Proc. of 10th Int. Conf. on Autonomous Agents and Multiagent Systems (AAMAS 2011), Tumer, Yolum, Sonenberg and Stone (eds.), May, 2-6, 2011, Taipei, Taiwan, pp. 727-734.

Copyright (C) 2011, International Foundation for Autonomous Agents and Multiagent Systems (www.ifaamas.org). All rights reserved. logics is the correspondence between their models and the game structures they are meant to reason about.

In particular, the connection between the semantics of Coalition Logic and games relies on Pauly's representation theorem [11] which states that playable effectivity functions correspond exactly to strategic games. Moreover, the correspondence has been used to obtain further results for CL: if the semantics can be defined equivalently in terms of strategic games and playable effectivity functions, they can be used interchangeably when proving properties of the logic. A similar remark applies to ATL and STIT, connected to Coalition Logic by a number of simulation results $[4,6,7]$.

The correspondence between strategic games and effectivity functions is important even without the logical context. Effectivity functions generalize basic models of cooperative game theory, whereas strategic games are models of noncooperative game theory. Pauly's result is relevant as it puts forward a characterization of strategic games in terms of coalitional games, therefore establishing a connection between the two families of game models.

In this paper, we show that the representation theorem is not correct as it stands. More precisely, we show that there are some playable effectivity functions with no corresponding strategic games. We point out where Pauly's proof of correspondence goes wrong, and we present examples of playable effectivity functions, for which no equivalent strategic games exist. Then, we define a more restricted class of effectivity functions, that we call truly playable, and we show that they correspond precisely to strategic games. We discuss several alternative characterizations of truly playable functions. Moreover, we present a construction that recovers the correspondence in the sense that it transforms any playable function into a truly playable one while preserving the power of most (but not all) coalitions. Finally, we discuss the ramifications for the above mentioned logics. On the one hand we show that the complete axiomatization of Coalition Logic from [11] is not affected if we change the class of models from playable to truly playable. On the other hand, we propose more expressive languages that can characterize the property of true playability, thus drawing a logical distinction with Pauly's playability.

\section{PRELIMINARIES}

\subsection{Strategic Games}

Strategic games are basic models of non-cooperative game theory [10]. After [11], we focus on abstract game forms, where the effect of strategic interaction between players is 
represented by abstract outcomes from a given set and players' preferences are not specified. For simplicity we refer to them as strategic games.

Definition 1 (Strategic game). A strategic game $G$ is a tuple $\left(N,\left\{\Sigma_{i} \mid i \in N\right\}, o, S\right)$ that consists of a nonempty finite set of players $N$, a nonempty set of strategies $\Sigma_{i}$ for each player $i \in N$, a nonempty set of outcomes $S$, and an outcome function o: $\prod_{i \in N} \Sigma_{i} \rightarrow S$ which associates an outcome with every strategy profile.

Additionally, we follow [11] and define coalitional strategies $\sigma_{C}$ in $G$ as tuples of individual strategies $\sigma_{i}$ for $i \in C$, i.e., $\Sigma_{C}=\prod_{i \in C} \Sigma_{i}$. Note that this definition allows for only one strategy $\sigma_{\emptyset}$ when $C=\emptyset$, namely the empty function.

\subsection{Effectivity Functions}

Effectivity functions have been introduced in cooperative game theory [9] to provide an abstract representation of the powers of coalitions to influence the outcome of the game.

Definition 2 (EFFECTIVITy Function). An effectivity function is a function $E: 2^{N} \rightarrow 2^{2^{S}}$, that associates a family of sets of states from $S$ with each set of players.

Intuitively, elements of $E(C)$ are choices available to coalition $C$ : if $X \in E(C)$ then by choosing $X$ the coalition $C$ can force the outcome of the game to be in $X$. Effectivity functions are usually required to satisfy additional properties, consistent with this interpretation.

Definition 3 (Playability [11]). An effectivity function $E$ is playable iff the following conditions hold:

Outcome Monotonicity $X \in E(C)$ and $X \subseteq Y$ implies $Y \in E(C)$;

N-maximality $\bar{X} \notin E(\emptyset)$ implies $X \in E(N)$;

Liveness $\emptyset \notin E(C)$;

Safety $S \in E(C)$;

Superadditivity if $C \cap D=\emptyset, X \in E(C)$ and $Y \in E(D)$, then $X \cap Y \in E(C \cup D)$.

Looking at playable effectivity functions, we can observe that their representation contains some redundancy. In particular, the fact that $E(C)$ is outcome monotonic suggests that one could succinctly represent it in terms of minimal sets, i.e., the elements of $E(C)$ that form an antichain under set inclusion. The nonmonotonic core, introduced in [11], is aimed at providing such a representation.

Definition 4 (Nonmonotonic CORE). Let $E$ be an effectivity function. The nonmonotonic core $E^{n c}(C)$ for $C \subseteq$ $N$ is the set of minimal sets in $E(C)$ :

$$
E^{n c}(C)=\{X \in E(C) \mid \neg \exists Y(Y \in E(C) \text { and } Y \subsetneq X)\} .
$$

We will show in Section 3.1 that not all sets in an effectivity function need to contain a set from the nonmonotonic core. Thus, $E^{n c}$ does not always behave well as a representation of the effectivity function, unless it is "complete" in the following sense.

Definition 5 (COMPlete nonmonotonic CORE). The nonmonotonic core $E^{n c}(C)$ is complete iff for every $X \in$ $E(C)$ there exists $Y \in E^{n c}(C)$ such that $Y \subseteq X$.
The nonmonotonic core of the empty coalition is of particular interest to us. For it, the following holds.

Proposition 1. For every playable effectivity function E:

1. $E(\emptyset)$ is a filter.

2. $E^{n c}(\emptyset)$ is either empty or a singleton.

Proof. (1) $E(\emptyset)$ is non-empty by Safety; closed under supersets by Outcome Monotonicity, and under intersections by Superadditivity (with respect to the empty coalition).

(2) Suppose $E^{n c}(\emptyset)$ is non-empty, and let $X, Y \in E^{n c}(\emptyset)$. Then, coalition $\emptyset$ is effective for each of $X$ and $Y$, hence, by superadditivity, it is effective for $X \cap Y$. By the definition of $E^{n c}(\emptyset)$, it follows that $X=X \cap Y=Y$.

Each strategic game $\mathcal{G}$ can be canonically associated with an effectivity function, called the $\alpha$-effectivity function of $\mathcal{G}$ and denoted with $E_{G}^{\alpha}$.

Definition 6 ( $\alpha$-Effectivity in Strategic Games). For a strategic game $G$ the $\alpha$-effectivity function $E_{G}^{\alpha}: 2^{N} \rightarrow$ $2^{2^{S}}$ is defined as follows: $X \in E_{G}^{\alpha}(C)$ if and only if there exists $\sigma_{C}$ such that for all $\sigma_{\bar{C}}$ we have $o\left(\sigma_{C}, \sigma_{\bar{C}}\right) \in X$.

Proposition 2. For every $\alpha$-effectivity function $E_{G}^{\alpha}: 2^{N} \rightarrow 2^{2^{S}}$, the following hold:

1. The nonmonotonic core of $E_{G}^{\alpha}(\emptyset)$ is the singleton set $\{Z\}$ where $Z=\left\{\{x\} \in S \mid x=o\left(\sigma_{N}\right)\right.$ for some $\left.\sigma_{N}\right\}$.

2. $E_{G}^{\alpha}(\emptyset)$ is the principal ${ }^{1}$ filter generated by $Z$.

Proof. For both claims it suffices to observe that $Z \in$ $E_{G}^{\alpha}(\emptyset)$ and that $Z \subseteq U$ for every $U \in E_{G}^{\alpha}(\emptyset)$. Therefore, $E^{n c}(\emptyset)=\{Z\}$ for $E=E_{G}^{\alpha}$ and $E_{G}^{\alpha}(\emptyset)$ is the principal filter generated by $Z$.

\section{PROBLEM WITH CORRESPONDENCE}

In this section we show that playability is not sufficient to make effectivity functions correspond to strategic games.

\subsection{Counterexample to Pauly's Representation Theorem}

Theorem 3 (Pauly's Representation Theorem [11]). $A$ coalitional effectivity function $E \alpha$-corresponds to a strategic game if and only if $E$ is playable.

Thus, the theorem states that every playable effectivity function is equal to the $\alpha$-effectivity function of some game (Pauly calls this equivalence relation $\alpha$-correspondence), and that each game has an $\alpha$-effectivity function that is playable. While the latter is true, the former turns out incorrect.

Proposition 4. There is a playable effectivity function $E$ for which $E \neq E_{G}^{\alpha}$ for all strategic games $G$.

Proof. Consider an effectivity function $E$ ranging on a set $N$ consisting of a single player $a$ and on the set of natural numbers $\mathbb{N}$ (i.e., $N=\{a\}, S=\mathbb{N}$ ), and defined as follows:

- $E(\{a\})=\{X \subseteq \mathbb{N} \mid X$ is infinite $\}$;

${ }^{1}$ Filter $F$ on domain $\Omega$ is principal iff there exists $X \subseteq \Omega$ such that $F$ is the set of all supersets of $X$. Then, $F$ is said to be generated by $X$. 


$$
\text { - } E(\emptyset)=\{X \subseteq \mathbb{N} \mid \bar{X} \text { is finite }\} \text {. }
$$

We claim that $E$ is playable and that it does not correspond to any strategic game. Let us first verify the playability conditions. Outcome monotonicity, N-maximality, liveness and safety are straightforward to check. For superadditivity, notice that we have only two cases to verify:

1. $C=\{a\}, D=\emptyset$. Superadditivity holds here because intersection of an infinite and a cofinite set is infinite.

2. $C=\emptyset, D=\emptyset$. Superadditivity in this case holds because intersection of two cofinite sets is cofinite.

On the other hand, $E^{n c}(\emptyset)=\emptyset$ because there are no minimal cofinite sets. This implies, by Proposition 2, that $E \neq E_{G}^{\alpha}$ for all strategic games $G$.

\subsection{Tracing the Problem}

Below, we summarize the relevant part of the proof of Theorem 2.27 from [11], and show where it goes wrong. We outline the construction of a strategic game $\mathcal{G}$ given an effectivity function $E$ (Steps 1-4); then, the argument supposed to show that $E \alpha$-corresponds to $\mathcal{G}$ (Steps 5-6).

Step 1: the players and the domain remain the same. The game $\mathcal{G}=\left(N, S, \Sigma_{i}, o\right)$ inherits the set of outcomes and the set of players as in the effectivity function $E$.

Step 2: coalitions choose a set from their effectivity function. Now, a family of functions is defined:

$F_{i}=\left\{f_{i}: \mathcal{C}_{i} \rightarrow 2^{S} \mid\right.$ for all $C$ we have that $\left.f_{i}(C) \in E(C)\right\}$

where $\mathcal{C}_{i}=\{C \subseteq N \mid i \in C\}$. Each function $f_{i}$ assigns choices to all coalitions of which $i$ is a member. $F_{i}$ simply collects all such assignments.

Step 3: coalitions are partitioned according to their choices. Let $f=\left(f_{i}\right)_{i \in N}, f_{i} \in F_{i}$, be a tuple of such assignments, one per player. The next step is to define the set $P_{\infty}(f)$ which results from iterative partitioning of the set of players in the coarsest possible way such that players in the same partition are assigned same coalitional choices:

$$
\begin{aligned}
P_{0}(f) & =\langle N\rangle \\
P_{1}(f) & =P(f, N)=\left\langle C_{1}^{1}, \ldots, C_{k_{1}}^{1}\right\rangle \\
P_{2}(f) & =\left\langle P\left(f, C_{1}^{1}\right), \ldots, P\left(f, C_{k_{1}}^{1}\right)\right\rangle=\left\langle C_{2}^{2}, \ldots, C_{k_{2}}^{2}\right\rangle \\
& \cdots \\
P_{\infty}(f) & =P_{r}(f) \text { such that } P_{i}(f)=P_{i+1}(f) \text { for all } i \geq r,
\end{aligned}
$$

where each $P(f, C)$ returns the coarsest partitioning $\left\langle C_{1}, \ldots, C_{m}\right\rangle$ of coalition $C$ such that for all $l \leq m$ and for all $i, j \in C_{l}$ it holds that $f_{i}(C)=f_{j}(C)$.

Step 4: an outcome is chosen in the intersection of coalitional choices. Strategies and outcome function are defined as follows. Each player in $N$ is given a set of strategies of the form $\left(f_{i}, t_{i}, h_{i}\right)$ where $f_{i} \in F_{i}$ is an assignment of coalitional choices for player $i$ (see Step 2), $t_{i}$ is a player (possibly different from $i$ ), and $h_{i}: 2^{S} \backslash \emptyset \rightarrow S$ is a selector function that picks up an arbitrary element from each nonempty subset of $S$.

The outcome of strategy $\sigma_{N}$ is now defined as:

$$
o\left(\sigma_{N}\right)=h_{i_{0}}(\mathcal{G}(f)), \quad \mathcal{G}(f)=\bigcap_{l=1}^{k} f\left(C_{l}\right),
$$

where $i_{0}$ is a uniquely chosen player, $h_{i_{0}}$ is the outcome selector from $i_{0}$ 's strategy, and $C_{l}$ is one of the $k$ coalitions in $P_{\infty}(f)$.

This concludes the construction of a game $\mathcal{G}$ which should $\alpha$-correspond to the effectivity function $E$. Steps $5-6$ are supposed to prove that $E=E_{\mathcal{G}}^{\alpha}$.

Step 5: choices are not removed by the construction. First, an attempt to prove $E(C) \subseteq E_{\mathcal{G}}^{\alpha}(C)$ for arbitrary coalition $C$ is presented [11, p.29]:

For the inclusion from left to right, assume that $X \in E(C)$. Choose any $C$-strategy $\sigma_{C}=\left(f_{i}, t_{i}, h_{i}\right)_{i \in C}$ such that for all $i \in C$ and for all $C^{\prime} \supseteq C$ we have $f_{i}\left(C^{\prime}\right)=X .\left(^{*}\right)$ By coalition monotonicity, such $f_{i}$ exists. $\left.{ }^{* *}\right)$ Take now any $\bar{C}$-strategy, $\sigma_{\bar{C}}=$ $\left(f_{i}, t_{i}, h_{i}\right)_{i \in \bar{C}}$. We need to show that $o\left(\sigma_{C}, \sigma_{\bar{C}}\right) \in$ $X$. To see this, note that $C$ must be a subset of one of the partitions $C_{l}$ in $P_{\infty}(f)$. Hence, $o\left(\sigma_{N}\right)=h_{i_{0}}(\mathcal{G}(f))=h_{i_{0}} \bigcap_{l=1}^{k} f\left(C_{l}\right) \in X$.

The deduction of the last sentence is where the proof goes wrong. The problem is that, for $C=\emptyset$, the only available strategy is the empty strategy $\sigma_{\emptyset}$ which vacuously satisfies condition $(*)$. And, for any agent $i$, a choice assignment $f_{i}$ satisfying the condition must exist. However, there is no guarantee that any $i$ will indeed choose $f_{i}$ in its strategy since the coalition $C$ for which we can fix its strategy does not include any players. In consequence, one cannot deduce that $h_{i_{0}}\left(\bigcap_{l=1}^{k} f\left(C_{l}\right)\right) \in X$; this could be only concluded if the intersection contains at least one player whose choice $f_{i}\left(C_{l}\right)$ is $X$ (or a subset of $X$ ).

Another case where the reasoning fails is $C=N$. Consider a state space $S$ with $x \in S$, and an effectivity function $E$ such that $\{x\} \notin E(N)$. Now, let strategy profile $\sigma_{N}$ consist of $\sigma_{i}=\left(f_{i}, t_{i}, h_{i}\right)$ where everybody assumes choosing the whole state space in all circumstances (i.e., $f_{i}(C)=S$ for all $i$ and $C$ ) and applies the same selector $h_{i}$ such that $h_{i}(S)=$ $x$. Now we get that $o\left(\sigma_{N}\right)=x$, so $\{x\} \in E_{\mathcal{G}}^{\alpha}(N)$, and hence $E(N) \neq E_{\mathcal{G}}^{\alpha}(N)$.

Step 6: choices are not added by the construction. The proof of the other direction $\left(E_{\mathcal{G}}^{\alpha}(C) \subseteq E(C)\right)$ fails too, because in order to establish the inclusion for $C=N$, it is reduced to inclusion in step 5 for $C=\emptyset$, and we have just shown that it does not necessarily hold.

This concludes our analysis of the proof of Pauly's representation theorem in [11]. We consider it important for two reasons. First, we have identified precisely what was wrong with the construction of the proof. Second, we will reuse the sound parts of the original construction when proving a revised version of the correspondence in Section 4.2 and to obtain some additional results in Section 4.4.

\section{TRULY PLAYABLE EFFECTIVITY FUNCTIONS}

In this section we introduce an additional constraint on playable effectivity functions, that will enable us to restore the correspondence with strategic games in in Section 4.2.

\subsection{Characterizing True Playability}

Definition 7. An effectivity function $E$ is truly playable iff it is playable and $E(\emptyset)$ has a complete nonmonotonic core. 
Several equivalent characterizations of truly playable effectivity functions are given in Proposition 5. For one of them, we will need the additional notion of a crown. Intuitively, an effectivity function is a crown if every choice of the agents in the grand coalition includes at least one state that the grand coalition can enforce precisely.

DEFINITION 8. An effectivity function $E$ is a crown iff $X \in E(N)$ implies $\{x\} \in E(N)$ for some $x \in X$.

Proposition 5. The following are equivalent for every playable effectivity function $E: 2^{N} \rightarrow 2^{2^{S}}$.

1. E is truly playable.

2. $E(\emptyset)$ has a non-empty nonmonotonic core.

3. $E^{n c}(\emptyset)$ is a singleton and $E(\emptyset)$ is a principal filter, generated by $E^{n c}(\emptyset)$.

4. $E$ is a crown.

Proof. (1) $\Rightarrow(2)$ : immediate, by safety.

$(2) \Rightarrow(3)$ : Let $Z \in E^{n c}(\emptyset)$ and let $X \in E(\emptyset)$. Then, by superadditivity, $Z \cap X \in E(\emptyset)$, and $Z \cap X \subseteq Z$, hence $Z \cap X=Z$ by definition of $E^{n c}(\emptyset)$. Thus, $Z \subseteq X$. So, $E(\emptyset)$ is the principal filter generated by $Z$, hence $E^{n c}(\emptyset)=\{Z\}$.

$(3) \Rightarrow(1)$ : immediate from the definitions.

$(3) \Rightarrow(4)$ : Let $E^{n c}(\emptyset)=\{Z\}$ and suppose $\{x\} \notin E(N)$ for all $x \in X$ for some $X \in E(\emptyset)$. Then, by N-maximality, $S \backslash\{x\} \in E(\emptyset)$, i.e. $Z \subseteq S \backslash\{x\}$ for every $x \in X$. Then $Z \subseteq S \backslash X$, hence $S \backslash X \in E(\emptyset)$. Therefore, $X \notin E(N)$ by superaditivity and liveness. By contraposition, $E$ is a crown.

$(4) \Rightarrow(3)$ : Let $Z=\{z \mid\{z\} \in E(N)\}$ and let $X \in E(\emptyset)$. Take any $z \in Z$, which is nonempty by liveness and the fact that $E$ is a crown. By superadditivity we obtain that $\{z\} \cap X \in E(\emptyset)$, hence $z \in X$ by liveness. Thus, $Z \subseteq X$. Moreover, $Z \in E(\emptyset)$, for else $S \backslash Z \in E(N)$ by N-maximality, hence $\{x\} \in E(N)$ for some $x \in S \backslash Z$, which contradicts the definition of $Z$. Therefore, $E(\emptyset)$ is the principal filter generated by $Z$, hence $E^{n c}(\emptyset)=\{Z\}$.

COROLlary 6. Every playable effectivity function $E: 2^{N} \rightarrow 2^{2^{S}}$ on a finite domain $S$ is truly playable.

Proof. Straightforward, by Proposition 5.3 and the fact that every filter on a finite set is principal.

\subsection{Truly Playable Functions Correspond to Strategic Games}

The proof of Theorem 2.27 from [11] fails when we consider the effectivity function of the empty coalition or the grand coalition. However the proof is correct for the other cases. We will now show that the additional condition of true playability yields correctness of the original construction from [11].

ThEOREM 7. A coalitional effectivity function $E \alpha$-corresponds to a strategic game if and only if $E$ is truly playable.

Proof. By Propositions 2 and 5, for any strategic game $\mathcal{G}$ its $\alpha$-effectivity function $E_{\mathcal{G}}^{\alpha}$ is truly playable.

For the other direction, given a truly playable effectivity function $E$, we slightly change Pauly's procedure outlined in Section 3.2 (Steps 1-4). We impose an additional constraint on players' strategies $\sigma_{i}=\left(f_{i}, t_{i}, h_{i}\right)$, namely, we require that $h_{i}(X)=x$ for some $\{x\} \in E(N)$. In other words, the selector functions only select the "jewels" in the crown. Note that for $C \notin\{\emptyset, N\}$ the new procedure yields game $\mathcal{G}^{\prime}$ with exactly the same $E^{\alpha}(C)$ as the original construction $\mathcal{G}$ from [11] (we omit the proof due to lack of space). It remains now to show that the procedure constructs a strategic game $\mathcal{G}$ such that $E(C)=E_{\mathcal{G}}^{\alpha}(C)$ for all $C \subseteq N$, that is, to show that steps 5 and 6 work well in truly playable structures.

Ad. Step 5. We show that $E(C) \subseteq E_{\mathcal{G}}^{\alpha}(C)$ for $C=\emptyset$ and $C=N$, the only cases in which the original proof failed.

Assume that $X \in E(\emptyset)$. We need to prove that $X \in$ $E_{\mathcal{G}}^{\alpha}(\emptyset)$. By true playability we know that there exists $Y \in$ $E^{n c}(\emptyset)$ such that $Y \subseteq X$. By Proposition $5, E^{n c}(\emptyset)=$ $\{Y\}$ and $E(\emptyset)=\{Z \mid Y \subseteq Z\}$. We will show now that $Y=\{x \mid\{x\} \in E(N)\}\left(^{*}\right)$. First, suppose that $x \in Y$ and $\{x\} \notin E(N)$, then by $N$-maximality $S \backslash\{x\} \in E(\emptyset)$, a contradiction. Second, let $\{x\} \in E(N)$ and $x \notin Y$, then by superadditivity $\emptyset \in E(N)$ which contradicts liveness.

Now, consider any strategy profile $\sigma_{N}$. We have $o\left(\sigma_{N}\right)=$ $h_{i_{0}}\left(\bigcap_{l=1}^{k} f\left(C_{l}\right)\right) \in Y$ because every $h_{i}$ returns only elements in $Y$ by construction.

For the case $C=N$, assume that $X \in E(N)$. We need to prove that $X \in E_{\mathcal{G}}^{\alpha}(N)$. By true playability, there exists $x \in X$ such that $\{x\} \in E(N)$. Now, let $\sigma_{N}$ consist of strategies $\sigma_{i}=\left(f_{i}, t_{i}, h_{i}\right)$ such that $f_{i}(N)=\{x\}$ for every $i$. It is easy to see that $o\left(\sigma_{N}\right)=x$, and hence $\{x\} \in E_{\mathcal{G}}^{\alpha}(N)$. Thus, $X \in E_{\mathcal{G}}^{\alpha}(N)$ because $E_{\mathcal{G}}^{\alpha}(N)$ is closed under supersets.

Ad. Step 6. Dually to Step 5, we show that $E_{\mathcal{G}}^{\alpha}(C) \subseteq$ $E(C)$. That is, assuming $X \notin E(C)$ we show that $X \notin$ $E_{\mathcal{G}}^{\alpha}(N)$. We do it by a slight modification of the original proof from [11].

Suppose first that $C=N$. Then, $\bar{X} \in E(\emptyset)$ by $N$ maximality, and by Step 5 we have $\bar{X} \in E_{\mathcal{G}}^{\alpha}(\emptyset)$. Since $E_{\mathcal{G}}^{\alpha}$ is truly playable, we have also that $X \notin E_{\mathcal{G}}^{\alpha}(N)$.

Assume now that $C \neq N$, and let $j_{0} \in \bar{C}$. Let $\sigma_{C}$ be any strategy for coalition $C$. We must show that there is a strategy $\sigma_{\bar{C}}$ such that $o\left(\sigma_{C}, \sigma_{\bar{C}}\right) \notin X$. To show this, we take $\sigma_{\bar{C}}=\left(f_{i}, t_{i}, h_{i}\right)_{i \in \bar{C}}$ such that for all $C^{\prime} \supseteq \bar{C}$ and for all $i \in \bar{C}$ we have $f_{i}\left(C^{\prime}\right)=S$. We also choose $t_{j_{0}}$ such that $\left(\left(t_{1}+\ldots+t_{n}\right) \bmod n\right)+1=j_{0}$. Note that $\bar{C}$ must be a subset of one of the partitions $C_{l}$ in $P_{\infty}(f)$, say $C_{l_{0}}$. Moreover, there must be a partitioning $\left\langle C_{1}, \ldots, C_{k}\right\rangle$ of $N \backslash C_{l_{0}}$ such that $\mathcal{G}(f)=f\left(C_{l_{0}}\right) \cap \bigcap_{l=1}^{k} f\left(C_{l}\right)=\bigcap_{l=1}^{k} f\left(C_{l}\right)$. Since $f\left(C_{l}\right) \in$ $E\left(C_{l}\right)$ we get that $\mathcal{G}(f) \in E(N) \backslash C_{l_{0}}$ by superadditivity. By coalition-monotonicity and the fact that $N \backslash C_{l_{0}} \subseteq C$, we also have $\mathcal{G}(f) \in E(C)$. Finally, by $(*)$ and superadditivity we obtain $\mathcal{G}(f) \cap\{x \mid\{x\} \in E(N)\} \in E(C)$.

Since $X \notin E(C)$ and $E(C)$ is closed under supersets, it must hold that $\mathcal{G}(f) \cap\{x \mid\{x\} \in E(N)\} \nsubseteq X$. Thus, there is some $s_{0} \in S$ such that: $s_{0} \in \mathcal{G}(f),\left\{s_{0}\right\} \in E(N)$, and $s_{0} \notin X$. Now we fix $h_{j_{0}}$ so that $h_{j_{0}}(\mathcal{G}(f))=s_{0}$. Then, $o\left(\sigma_{C}, \sigma_{\bar{C}}\right)=h_{j_{0}}(\mathcal{G}(f))=s_{0} \notin X$ which concludes the proof.

\subsection{Non-Truly Playable Structures}

In this section we focus on the class of playable but not truly playable effectivity functions, hereafter called "nontruly playable". Non-truly playable effectivity functions have a simple abstract characterization, following from Proposition 5:

Proposition 8. Effectivity function $E: 2^{N} \rightarrow 2^{2^{S}}$ is non-truly playable if and only if it is playable and $E(\emptyset)$ is a non-principal filter. 
To see a more generic class of examples, consider an infinite domain $S$, and let $\mathcal{F}$ be any non-principal filter on $S$. Then we define an effectivity function $E_{\mathcal{F}}$ on $S$ as follows.

- $E_{\mathcal{F}}(\emptyset)=\mathcal{F}$.

- $E_{\mathcal{F}}(N)=\{X \mid \bar{X} \notin \mathcal{F}\}$

- For each $C$ with $\emptyset \subsetneq C \subsetneq N$ take $E_{\mathcal{F}}(C)$ to be any set of sets such that $E_{\mathcal{F}}(\emptyset) \subseteq E_{\mathcal{F}}(C) \subseteq E_{\mathcal{F}}(N)$ that is closed under outcome monotonicity and that are pairwise closed under regularity and superadditivity.

Proposition 9. $E_{\mathcal{F}}$ is playable but not truly playable.

We omit the proof due to lack of space.

\subsection{From Playable to Truly Playable Effectiv- ity Functions}

In this section we show that one can reconstruct a nontruly playable effectivity function into a truly playable one with "minimal" modifications. To do so, we interpret choices of the grand coalition containing multiple outcome states as ones that involve inherent nondeterminism. That is, we interpret $\left\{x_{1}, x_{2}, \ldots\right\} \in E(N)$ as a choice where no agent has control over which state out of $x_{1}, x_{2}, \ldots$ will become the outcome; as a consequence any of these states can possibly be encountered in the next moment. Under such assumption, it is possible to recover true playability by a simple extension of Pauly's procedure. The extension consists in adding an extra player $\mathbf{d}$ (the "decider") who settles the nondeterminism and decides which of $x_{1}, x_{2}, \ldots$ is going to become the next state.

Proposition 10. Let $E: 2^{N} \rightarrow 2^{2^{S}}$ be a playable effectivity function. There exists a truly playable effectivity function $E^{\prime}: 2^{N \cup\{\mathbf{d}\}} \rightarrow 2^{2^{S}}$ with additional player $\mathbf{d} \notin N$, such that:

- $E^{\prime}(C)=E(C)$ for every $C \subseteq N, C \neq \emptyset$,

- $E^{\prime}(\emptyset)=\{S\}$, and

- $E^{\prime}(N \cup\{\mathbf{d}\})=2^{S} \backslash\{\emptyset\}$.

Proof. Given a playable $E$, we construct a strategic game whose $\alpha$-effectivity function satisfies the properties above. Then, existence of a truly playable effectivity function follows immediately. The idea is to take the construction from the proof of Theorem 2.27 in [11] and reassign selection of the outcome state to the additional player $\mathbf{d}$.

Let $h: 2^{S} \backslash\{\emptyset\} \rightarrow S$ be any selector function that selects an arbitrary element from the argument set. In our case, $h$ will designate the "default" outcome for each subset of $S$. Now, the game $\mathcal{G}$ is constructed as follows:

- $N^{\prime}=N \cup\{\mathbf{d}\}$;

- The strategies of each player $i \neq \mathbf{d}$ are simply the player's assignments of coalitional choice, i.e., $\Sigma_{i}=F_{i}$, as in section 3.2 ;

- The strategies of $\mathbf{d}$ are state selections: $\Sigma_{\mathbf{d}}=S$;

- The transition function is based on the same partitioning of $N$ as before, that yields $\left\langle C_{1}, \ldots, C_{k}\right\rangle$. Then, the game proceeds to the state selected by the decider if his choice is consistent with the choices of the others, otherwise it proceeds to the appropriate "default" outcome:

$$
o\left(\sigma_{N}, s\right)= \begin{cases}s & \text { if } s \in \bigcap_{i=1}^{k} f\left(C_{l}\right) \\ h\left(\bigcap_{i=1}^{k} f\left(C_{l}\right)\right) & \text { else. }\end{cases}
$$

Now, it is easy to see that for every $\emptyset \subsetneq C \subsetneq N$ indeed $E_{\mathcal{G}}^{\alpha}(C)=E(C)$ because that was the case in the original construction, and the only difference now is that $\mathbf{d}$ "took over" the selection of a state in $\bigcap_{i=1}^{k} f\left(C_{l}\right)$ from a collective choice of $N$. For $C=N$, we also have $E_{\mathcal{G}}^{\alpha}(N)=E(N)$ since for every $\sigma_{N}$ we get by superadditivity that $\bigcap_{i=1}^{k} f\left(C_{l}\right) \in E(N)$, and every state from the intersection can be potentially selected by d. Moreover, $\{s\} \in E_{\mathcal{G}}^{\alpha}(N \cup\{\mathbf{d}\})$ for every $s \in S$ because $\{s\}$ is enforced by $\sigma_{N \cup\{\mathbf{d}\}}=\left\langle f_{1}, \ldots, f_{|N|}, s\right\rangle$ such that $f_{i}=S$ for all $i \in N$. Thus, by outcome monotonicity, $E_{\mathcal{G}}^{\alpha}(N \cup\{\mathbf{d}\})=2^{S} \backslash\{\emptyset\}$. Finally, by true playability of $E_{\mathcal{G}}^{\alpha}$, we have $E_{\mathcal{G}}^{\alpha}(\emptyset)=\left\{\left\{s \mid\{s\} \in E_{\mathcal{G}}^{\alpha}(N \cup\{\mathbf{d}\})\right\}\right\}=\{S\}$. We observe additionally that $E_{\mathcal{G}}^{\alpha}(\mathbf{d})=\{\{s\} \cup\{h(X) \mid X \in$ $E_{G}^{\alpha}(\mathbf{d})$ and $\left.\left.s \notin X\right\} \mid s \in S\right\}$.

\section{LOGICS AND TRUE PLAYABILITY}

In this section, we investigate the impact of true playability on logics of coalitional ability. We begin by indicating that the validities of Coalition Logic do not change if we restrict models to truly playable. As a consequence, CL (and even ATL) cannot distinguish between playable and truly playable models. Then, we discuss two extensions of CL that can discern the two classes of structures.

For preliminaries on modal logic see e.g. $[5,3]$.

\subsection{Ramifications for $\mathbf{C L}$}

We recall from [11] that the models of Coalition Logic (also called coalition models ) are neighborhood models of the type $M=(W, E, V)$ consisting of a set of states $W$, a dynamic effectivity function $E: W \rightarrow\left(2^{N} \rightarrow 2^{2^{W}}\right)$ and a valuation function $V: W \rightarrow 2^{P}$ ranging over a countable set of atomic propositions $P$. A coalitional frame is a coalition model minus the valuation. A model (resp. frame) is playable iff it includes only playable effectivity functions at each $w \in W$, and truly playable iff it includes only truly playable functions at each $w \in W$. The operator $[C]$ is interpreted as follows:

$$
M, w \models[C] \phi \text { if and only if } \phi^{M} \in E(w)(C),
$$

where $\phi^{M}$ is the set $\{v \in W \mid M, v \models \phi\}$. Formula $\varphi$ is valid in model $M(M \mid \varphi)$ if and only if it holds in every state in $M ; \varphi$ is valid in frame $F(F \models \varphi)$ if and only if it is valid in every model based on $F$. We extend these notions to classes of models and frames in the obvious way.

We note that the problem with Pauly's Representation Theorem has no repercussions on the semantics of CL and the soundness/completeness results for that logic. Let us formally define Play to be the class of playable coalition models, and TrulyPlay as the class of truly playable models. Since TrulyPlay $\subsetneq$ Play, every CL formula valid in Play is valid in TrulyPlay, too. The converse follows from the finite model property of CL with respect to Play [11] and the fact that it coincides with TrulyPlay on finite models.

COROLlary 11. The axiomatization of CL from [11] is sound and complete wrt truly playable coalition models, and hence, also with respect to strategic game models.

Furthermore, the semantics based on effectivity functions can be extended to ATL (see. [6]; also, cf. [11] for the fragment of ATL without "until", called Extended $C L$ ). Again, it can be shown that Play and TrulyPlay determine the same 
sets of validities for ATL, by checking the soundness of the axiomatization for ATL given in [7] for Play, and using the completeness result for ATL with respect to strategic game models (equivalently, TrulyPlay) proved in the same paper.

\subsection{CL with Infinite Disjunctions}

One possible extension of CL that can tell apart the classes Play and TrulyPlay involves infinite disjunctions of formulas. The idea is that in truly playable models, every choice of the grand coalition can be narrowed down to a singleton. The infinitary disjunction $\bigvee_{i \in \mathcal{I}}$ for a set of indices $\mathcal{I}$ has the natural interpretation:

$$
M, w \models \bigvee_{i \in \mathcal{I}} \phi_{i} \text { if and only if } M, w \models \phi_{i} \text { for some } i \in \mathcal{I} \text {. }
$$

Proposition 12. For any cardinal number ${ }^{2} \kappa$, let Play ${ }_{\kappa}$ (resp. TrulyPlay ${ }_{\kappa}$ ) denote the class of playable (resp. truly playable) coalition models with the domain of outcomes $W$ of cardinality at most $\kappa$ and let $\left\{p_{\iota}\right\}_{\llcorner\in \kappa}$ be a set of different propositional letters.

$$
\begin{aligned}
& \text { 1. } \text { Play }_{\kappa} \not \models[N] \bigvee_{\iota \in \kappa} p_{\iota} \leftrightarrow \bigvee_{\iota \in \kappa}[N] p_{\iota} ; \\
& \text { 2. TrulyPlay } \models[N] \bigvee_{\iota \in \kappa} p_{\iota} \leftrightarrow \bigvee_{\iota \in \kappa}[N] p_{\iota}
\end{aligned}
$$

Proof. For (1) simply check the example in Section 3.1 with the set $S$ being $\kappa$ and every state $\iota$ associated with a designating atomic proposition $p_{\iota}$. Claim (2) follows from Proposition 5.

\subsection{CL with “Outcome Selector” Modality}

Adding infinitary operators to a logical language makes its practical applicability problematic. Here we propose another (in fact, simpler) extension of CL, by adding a new normal modality $\langle\mathcal{O}\rangle$, with a dual $[\mathcal{O}]$, called "outcome selector". The informal reading of $\langle\mathcal{O}\rangle \phi$ should be "there is an outcome state, enforceable by the grand coalition and satisfying $\phi "$. In order to define the semantics of $\langle\mathcal{O}\rangle$ in the usual semantic way, we first expand coalition models to what we call extended coalition models with an additional "outcome enforceability" relation $R$. Later we will use axioms to impose the right behavior of $R$.

Definition 9 (Extended COALition frames). An extended (playable) coalition frame is a neighbourhood frame $F=(W, E, R)$ where $W$ is a set of outcomes, $E$ a playable effectivity function, $R$ a binary relation on $W$.

An extended coalition model is an extended coalition frame endowed with a valuation function. Given an extended coalition model $M=(W, E, R, V)$, the modality $\langle\mathcal{O}\rangle$ is interpreted as follows.

$M, w \models\langle\mathcal{O}\rangle \phi$ if and only if $w R s$ and $M, s \models \phi$.

That is, $\langle\mathcal{O}\rangle$ has standard Kripke semantics with respect to the outcome enforceability relation $R$. Note that extended coalition models do not require any interaction between the effectivity function and the relation $R$. However, given the intuitive reading of the relation $R$, the interaction suggests itself, and the following definition account for that.

DEFINITION 10 (STANDARD COALITION FRAMES). $A$ standard coalition frame is an extended coalition frame such that, for all $w, v \in W$, we have $w R v$ if and only if $\{v\} \in E(w)(N)$.

${ }^{2}$ We regard cardinals as (special) ordinals in von Neumann sense: any ordinal is the set of all smaller ordinals.
A standard coalition model is a standard coalition frame with a valuation function. Depending on the properties of the underlying effectivity functions we call extended coalition frames and models playable or truly playable.

\subsection{Characterizing Standard Truly Playable Coalition Frames}

Proposition 13. An extended coalition frame $F$ is standard and truly playable if and only if $F \models[N] q \leftrightarrow\langle\mathcal{O}\rangle q$, for any atomic proposition $q$.

Proof. Left to right: Assume that $F$ is standard and truly playable. Assume first that $(F, V), w=[N] q$ for any $V$ and $w \in W$. By definition of $E$ we have that $q^{M} \in E(w)(N)$. As $F$ is truly playable there is $v \in q^{M}$ with $\{v\} \in E(w)(N)$. However $F$ is also standard so $w R v$. But this means that $(F, V), w \models\langle\mathcal{O}\rangle q$. Conversely, if $(F, V), w \models\langle\mathcal{O}\rangle q$ then $w R v$ for some $v \in q^{M}$. F being standard we have that $\{v\} \in E(w)(N)$. By outcome monotonicity $q^{M} \in E(w)(N)$, i.e. $(F, V), w \models[N] q$.

Right to left: Assume that $F=[N] q \leftrightarrow\langle\mathcal{O}\rangle q$. Let us first prove that $F$ is standard. Suppose $w R v$ for some $w, v \in W$. Let $V$ be a valuation that assigns the atom $q$ only to $v$. We have that $M, w \models\langle\mathcal{O}\rangle q$. Then, by the assumptions we also have $M, w \mid=[N] q$, which means that $\{v\} \in E(w)(N)$. Conversely, suppose now that $\{v\} \in E(w)(N)$. For the same valuation $V$ we must have that $(F, V), w=[N] q$ and by assumption that $\langle\mathcal{O}\rangle q$, which means that $w R v$. Thus, $F$ is standard. To prove that $F$ is truly playable, assume that for some $X \subseteq W, X \in E(w)(N)$ and let now $V$ be a valuation function such that $V(q)=X$. By definition of $E$ we have that $(F, V), w \models[N] q$, hence by assumption, that $(F, V), w \models\langle\mathcal{O}\rangle q$, which means that $w R v$ for some $v \in V(q)$. Then, $F$ being standard, $\{v\} \in E(w)(N)$.

\subsection{Standard Truly Playable Models: Axioms}

We propose the following axiomatic system TPCL for the class of standard truly playable coalition models TrulyPlay, extending Pauly's axiomatization of CL. The axioms include propositional tautologies plus the following schemes:

$$
\begin{aligned}
& \text { 1. }[N] \top \\
& \text { 2. } \neg[C] \perp \\
& \text { 3. } \neg[\emptyset] \phi \rightarrow[N] \neg \phi \\
& \text { 4. }[C] \phi \wedge[D] \psi \rightarrow[C \cup D](\phi \wedge \psi) \text { for any disjoint } C, D \subseteq N \\
& \text { 5. }[N] \phi \leftrightarrow\langle\mathcal{O}\rangle \phi \\
& \text { 6. }[\mathcal{O}](\phi \rightarrow \psi) \rightarrow([\mathcal{O}] \phi \rightarrow[\mathcal{O}] \psi) .
\end{aligned}
$$

The inference rules are: Modus Ponens, plus:

$$
\frac{\phi \rightarrow \psi}{[C] \phi \rightarrow[C] \psi}, \quad \text { and } \quad \frac{\phi}{[\mathcal{O}] \phi}
$$

REMARK. Axiom 5 seems to render the outcome modality $[\mathcal{O}]$ redundant. This, however, is not so, because the semantics of the modality $[N]$ is (monotonic) neighbourhood semantics, while the semantics of $[\mathcal{O}]$ is by default Kripke semantics. Relating these by Axiom 5 suffices to enforce the true playability of the underlying frames, as shown in Proposition 13. On the other hand, it is easy to show that the normality Axiom 6, as well as the necessitation rule for $[\mathcal{O}]$, are derivable from the rest. We have only added them to emphasize the fact that $[\mathcal{O}]$ is a normal modality.

The proof of the following claim is routine. 
Proposition 14. TPCL is sound for the class TrulyPlay: every formula derivable in TPCL is valid in TrulyPlay.

\subsection{Completeness for $T P C L$}

TheOREM 15 (COMPLETENEss THEOREM). Every formula consistent in TPCL is satisfiable in TrulyPlay. Consequently, the logic TPCL is complete for the class TrulyPlay.

We will prove the completeness, using canonical model construction followed by filtration for monotonic modal logics, partly using constructions from [5] and [11]. Thus, we will also obtain finite model property for TPCL. Here we only sketch the standard canonical model construction and refer the reader for further details to [5] and [11].

We start with a formula $\delta$ which is consistent in TPCL. By a well-known argument, it is contained in some maximal TPCL-consistent set. We take the set $W^{\mathcal{L}}$ of maximally consistent sets and define for every formula $\phi$ the proof set of $\phi$ as $\phi^{*}=\left\{s \in W^{\mathcal{L}} \mid \phi \in s\right\}$.

To shorten the notation we hereafter denote the logic TPCL by $\mathcal{L}$.

Definition 11 (CANONICAL MODEL). The canonical model for TPCL is $M^{\mathcal{L}}=\left(W^{\mathcal{L}}, E^{\mathcal{L}}, R^{\mathcal{L}}, V^{\mathcal{L}}\right)$ where:

- $w \in V^{\mathcal{L}}(p)$ if and only if $p \in w$;

- $X \in E^{\mathcal{L}}(w)(C)$ iff $\exists \psi^{*} \subseteq X:[C] \psi \in w, \quad$ for $C \neq N$

- $X \in E^{\mathcal{L}}(w)(C)$ iff $\forall \psi^{*}$ if $X \subseteq \psi^{*}$ then $[C] \psi \in w$, for $C=N$

- $w R^{\mathcal{L}} v$ iff $\forall \psi$, if $\psi \in v$ then $\langle\mathcal{O}\rangle \psi \in w$.

Some remarks:

- That $E^{\mathcal{L}}$ is playable and well-defined is proved in [11].

- The canonical relation for $N$ is defined in [11] in the following equivalent way: $X \in E^{\mathcal{L}}(w)(N)$ if and only if $[\emptyset] \psi \notin w$ for all $\psi^{*}$ such that $\psi^{*} \subseteq \bar{X}$. The equivalence follows easily from the fact that $\vdash_{\mathcal{L}}[N] \phi \leftrightarrow \neg[\emptyset] \neg \phi$.

Proposition 16 (Truth Lemma). For any $w \in W^{\mathcal{L}}$ we have that $M^{\mathcal{L}}, w \models \phi$ if and only if $\phi \in w$.

Proof. By induction on the length of $\phi$ : standard for atomic propositions, boolean formulas, and formulas of the form $\langle\mathcal{O}\rangle \psi$; proved in [11] for formulas of the form $[C] \psi$.

The canonical model is an extended coalition model, however it is neither standard nor truly playable. The reason for that is the fact that for all $\psi \in \mathcal{L}, \psi \in v$ implies that $[N] \psi \in$ $w$ is not sufficient to conclude that $\{v\} \in E^{\mathcal{L}}(w)(N)$ as states are not characterized by unique formulas of the language of $\mathcal{L}$. In order to obtain a standard and truly playable model satisfying the given $\mathcal{L}$-consistent formula $\delta$ we are going to filter the canonical model with the set $\Sigma(\delta)$, obtained by taking all subformulae of $\delta$ and closing under boolean operators. That set is finite up to propositional equivalence.

\section{Filtrations.}

First, we define a general notion of filtration for extended coalition models and then a special filtration construction that preserves playability. Filtrations of coalition models are introduced, e.g., in [8] for the purpose of axiomatizing Nashconsistent Coalition Logic. Here we only add the filtration for the relation corresponding to the modality $\langle\mathcal{O}\rangle$.

Let $M=(W, E, R, V)$ be an extended coalition model and $\Sigma$ a subformula-closed set of formulas over $\mathcal{L}$. The equivalence classes induced by $\Sigma$ on $M$ are defined as follows:

$v \equiv_{\Sigma} w \Leftrightarrow$ for all $\phi \in \Sigma: M, v \models \phi$ if and only if $M, w \mid=\phi$.
We denote the equivalence class to which $v$ belongs by $|v|$ and the set $\{|v| \mid v \in X\}$ by $|X|$ for any $v \in W$ and $X \subseteq W$.

Definition 12 (Filtration). Let $M=(W, E, R, V)$ be an extended coalition model and $\Sigma$ a subformula closed set of formulas over $\mathcal{L}$. A coalition model $M_{\Sigma}^{f}=\left(W_{\Sigma}^{f}, E_{\Sigma}^{f}, R_{\Sigma}^{f}, V_{\Sigma}^{f}\right)$ is a filtration of $M$ through $\Sigma$ whenever the following conditions are satisfied:

- $W_{\Sigma}^{f}=|W|$.

- For all $C \subseteq N$ and $\phi \in \Sigma, \phi^{M} \in E(w)(C)$ implies $\{|v| \mid M, v \models \phi\} \in E_{\Sigma}^{f}(|w|)(C)$.

- For all $C \subseteq N$ and $Y \subseteq|W|: \quad Y \in E_{\Sigma}^{f}(|w|)(C)$ implies that for all $\phi \in \Sigma$ if $\phi^{M} \subseteq\{v|| v \mid \in Y\}$ then $\phi^{M} \in E(w)(C)$.

- If $w R v$ then $|w| R|v|$.

- If $|w| R|v|$ then for all $\langle\mathcal{O}\rangle \phi \in \Sigma$, if $M, v \models \phi$ then $M, w \models$ $\langle\mathcal{O}\rangle \phi$.

- $V_{\Sigma}^{f}(p)=|V(p)|$ for all atoms $p \in \Sigma$.

The conditions above are needed to ensure the Filtration Lemma, as showed in [8] for the neighbourhood relations and e.g., in [5] for the binary relation.

Proposition 17 (Filtration Lemma). If $M_{\Sigma}^{f}=$ $\left(W_{\Sigma}^{f}, E_{\Sigma}^{f}, R_{\Sigma}^{f}, V_{\Sigma}^{f}\right)$ is a filtration of $M$ through $\Sigma$ then for all $\phi \in \Sigma$ we have that $M, w \models \phi$ if and only if $M_{\Sigma}^{f},|w| \models \phi$.

Definition 13 (Playable Filtration). Let $M=$ $(W, E, R, V)$ be an extended coalition model and $\Sigma(\delta)$ the boolean closure of the set of subformulas of $\delta$, such that $\delta \in \mathcal{L}$, the language of TPCL. A coalition model $M_{\Sigma(\delta)}^{F}=$ $\left(W_{\Sigma(\delta)}^{F}, E_{\Sigma(\delta)}^{F}, R_{\Sigma(\delta)}^{F}, V_{\Sigma(\delta)}^{F}\right)$ is a playable filtration of $M$ through $\Sigma(\delta)$ whenever the following conditions are satisfied:

- $W_{\Sigma(\delta)}^{F}=|W|$.

- For all $C \subsetneq N, C \neq N$, and $Y \subseteq|W|: Y \in E_{\Sigma(\delta)}^{F}(|w|)(C)$ if and only if there exists $\phi \in \Sigma(\delta)$ such that $\phi^{M} \subseteq\{v \mid$ $|v| \in Y\}$ and $\phi^{M} \in E(w)(C)$.

- For all $Y \subseteq|W|: Y \in E_{\Sigma(\delta)}^{F}(|w|)(N)$ if and only if $\bar{Y} \notin E_{\Sigma(\delta)}^{F}(|w|)(\emptyset)$.

- $|w| R_{\Sigma(\delta)}^{F}|v|$ if and only if there exists $w^{\prime} \in|w|, \exists v^{\prime} \in|v|$ such that $w^{\prime} R v^{\prime}$.

- $V_{\Sigma(\delta)}^{F}(p)=|V(p)|$ for all atoms $p \in \Sigma(\delta)$.

That $M_{\Sigma(\delta)}^{F}$ is a filtration in the sense of Definition 12 is proved in [8] for the coalitional modalities. We have added to that a minimal filtration for modality $\langle\mathcal{O}\rangle$. So $M_{\Sigma(\delta)}^{F}$ is a filtration in the sense of Definition 12. In [8] it is also shown that playability is preserved by that filtration and that every subset of $W_{\Sigma(\delta)}^{F}$ is definable by a formula of $\Sigma(\delta)$ as follows. First, for every state $|w| \in|W|$ we define

$$
\chi_{\Sigma(\delta)}^{F}(|w|):=\bigwedge\left\{\phi \in \Sigma(\delta)\left|M_{\Sigma(\delta)}^{F},\right| w \mid \models \phi\right\} .
$$

Then, for every $Y \subseteq|W|$ we put

$$
\chi_{\Sigma(\delta)}^{F}(Y):=\bigvee\left\{\chi_{\Sigma(\delta)}^{F}(|w|)|| w \mid \in Y\right\} .
$$


It is straightforward to show, using the filtration lemma, that for every $Y \subseteq|W|$ :

$$
M_{\Sigma(\delta)}^{F},|w|=\chi_{\Sigma(\delta)}^{F}(Y) \text { if and only if }|w| \in Y,
$$

that is, $\chi_{\Sigma(\delta)}^{F}(Y)$ indeed characterizes the set $y$ in $M_{\Sigma(\delta)}^{F}$.

Proposition 18. $M_{\Sigma(\delta)}^{\mathcal{L}, F}$ is standard and truly playable.

Proof. To prove that $M_{\Sigma(\delta)}^{\mathcal{L}, F}$ is standard we have to show that for each $w, v \in W,|v| R_{\Sigma(\delta)}^{\mathcal{L}, F}|w|$ if and only if $\{|v|\} \in$ $E_{\Sigma(\delta)}^{\mathcal{L}, F}(|w|)(N)$. From right to left it is straightforward. For the other direction, suppose $|v| R_{\Sigma(\delta)}^{\mathcal{L}, F}|w|$. Then $M_{\Sigma(\delta)}^{\mathcal{L}, F},|v| \models$ $\langle\mathcal{O}\rangle \chi_{\Sigma(\delta)}^{F}(|w|)$ by definition of $R_{\Sigma(\delta)}^{\mathcal{L}, F}$ and by the properties of filtrations. By the fact that $R_{\Sigma(\delta)}^{\mathcal{L}, F}$ is a minimal filtration we have that $\exists w^{\prime} \in|w|, \exists v^{\prime} \in|v|$ such that $v^{\prime} R^{\mathcal{L}} w^{\prime}$. By definition of $R^{\mathcal{L}}$ and the Truth Lemma we have that $M^{\mathcal{L}}, v^{\prime} \models\langle\mathcal{O}\rangle \chi_{\Sigma(\delta)}^{F}(|w|)$. By the axioms of $\mathcal{L}$ and the Truth Lemma we have $M^{\mathcal{L}}, v^{\prime} \models[N] \chi_{\Sigma(\delta)}^{F}(|w|)$, hence $M^{\mathcal{L}}, v^{\prime} \models$

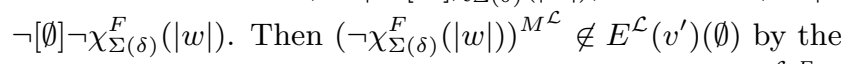
definition of $E^{\mathcal{L}}$. But, by Definition $12\left\{\left(\neg \chi_{\Sigma(\delta)}^{F}(|w|)\right)^{M_{\Sigma}^{\mathcal{L}, F}(\delta)}\right\} \notin$ $E_{\Sigma(\delta)}^{\mathcal{L}, F}(|v|)(\emptyset)$ and in turn $\left\{\left(\chi_{\Sigma(\delta)}^{F}(|w|)\right)^{M_{\Sigma(\delta)}^{\mathcal{L}, F}}\right\} \in E_{\Sigma(\delta)}^{\mathcal{L}, F}(|v|)(N)$. Recall now that $\left(\chi_{\Sigma(\delta)}^{F}(|w|)\right)^{M_{\Sigma(\delta)}^{\mathcal{L}, F}}=|w|$.

Now, to prove that $M_{\Sigma(\delta)}^{\mathcal{L}, F}$ is truly playable, assume $Y \in$ $E_{\Sigma(\delta)}^{\mathcal{L}, F}(|w|)(N)$. Then, $\left(\neg \chi_{\Sigma(\delta)}^{F}(Y)\right)^{M_{\Sigma(\delta)}^{\mathcal{L}, F}} \notin E^{\mathcal{L}}(w)(\emptyset)$ by the definition of filtration, which means that for all $\phi \in \Sigma(\delta)$, if $\left\{v|| v \mid \in\left(\neg \chi_{\Sigma(\delta)}^{F}(Y)\right)^{M_{\Sigma(\delta)}^{\mathcal{L}, F}}\right\} \subseteq \phi^{M}$ then $\phi^{M} \notin E^{\mathcal{L}}(w)(\emptyset)$.

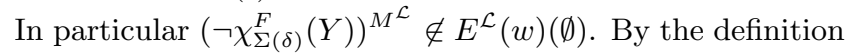
of $E^{\mathcal{L}}$ we have that $[\emptyset] \neg \chi_{\Sigma(\delta)}^{F}(Y) \notin w$ and by true playability that $\langle\mathcal{O}\rangle \chi_{\Sigma(\delta)}^{F}(Y) \in w$. By the definition of canonical relation for $\langle\mathcal{O}\rangle$ we have that there exists $v$ with $w R^{\mathcal{L}} v$ such that $\chi_{\Sigma(\delta)}^{F}(Y) \in v$. By definition of filtration $|w| R_{\Sigma(\delta)}^{\mathcal{L}, F}|v|$ and by the Filtration Lemma $M_{\Sigma(\delta)}^{\mathcal{L}, F},|v|=\chi_{\Sigma(\delta)}^{F}(Y)$. Finally, $\{|v|\} \in E_{\Sigma(\delta)}^{\mathcal{L}, F}(|w|)(N)$ since $M_{\Sigma(\delta)}^{\mathcal{L}, F}$ is standard.

This completes the proof of the Completeness theorem 15.

Corollary 19 (Finite Model Property). The logic TPCL has the finite model property with respect to the class of models TrulyPlay.

\section{CONCLUSIONS}

In this paper, we have revisited the correspondence between two classes of abstract game forms: strategic games from noncooperative game theory on one hand, and effectivity functions from cooperative game theory on the other. We consider our contribution as threefold. First, we have corrected a well-known and often used result from [11] relating strategic games and playable effectivity functions. We have shown that strategic games do not correspond to all playable functions, but to a strict subset of the class, which we call truly playable effectivity functions. Second, we have provided several abstract characterizations of truly playable functions. We have also shown that the remaining playable effectivity functions (that we call non-truly playable) are induced by non-principal filters, and hence only scenarios with infinitely many possible outcomes can fall in that class. Finally, we have pointed out that Coalition Logic and ATL are not expressive enough to characterize true playability. On the other hand, they can be extended in a relatively simple way to obtain such a characterization. To this purpose we have proposed an extension of Coalition Logic with a normal outcome selector modality that we have shown sufficient for axiomatic characterization of truly playable structures.

The importance of our work is mainly theoretical. Essentially, it implies that all the claims that have been proved using Pauly's correspondence between playable effectivity functions and games should be revisited and possibly reinterpreted in the light of the results presented here. Example of such issues, already addressed here, include: axiomatization for Coalition Logic in the class of multi-player game models, axiomatization of ATL in coalitional models, and the respective finite model properties. In practical terms, this also means that, whenever a decision procedure is built on those theoretical results, the designer should be aware of the correct correspondence between the two classes of game models, which is especially relevant for satisfiabilitychecking algorithms. Tableaux for extensions of Coalition Logic, like the one for a combination of CL and description logic $\mathcal{A L C}$ from [12], are examples of such procedures.

Acknowledgements. Wojciech Jamroga acknowledges the support of the FNR (National Research Fund) Luxembourg under project S-GAMES - C08/IS/03. Valentin Goranko acknowledges the support of the HYLOCORE project, funded by the Danish Natural Science Research Council.

\section{REFERENCES}

[1] R. Alur, T. A. Henzinger, and O. Kupferman. Alternating-time temporal logic. In W. P. de Roever, H. Langmaack, and A. Pnueli, editors, COMPOS, volume 1536 of Lecture Notes in Computer Science, pages 23-60. Springer, 1997.

[2] N. Belnap, M. Perloff, and M. Xu. Facing the Future: Agents and Choices in Our Indeterminist World. Oxford University Press, 2001.

[3] P. Blackburn, M. de Rijke, and Y. Venema. Modal Logic. Cambridge Tracts in Theoretical Computer Science, 2001.

[4] J. Broersen, A. Herzig, and N. Troquard. A normal simulation of coalition logic and an epistemic extension. In D. Samet, editor, Proceedings Theoretical Aspects Rationality and Knowledge (TARKXI), Brussels, pages 92-101. ACM Digital Library, 2007.

[5] B. Chellas. Modal Logic: an Introduction. Cambridge University Press, 1980.

[6] V. Goranko and W. Jamroga. Comparing semantics of logics for multi-agent systems. Synthese, 139(2):241-280, 2004.

[7] V. Goranko and G. van Drimmelen. Complete axiomatization and decidability of alternating-time temporal logic. Theor. Comput. Sci., 353(1-3):93-117, 2006.

[8] H. H. Hansen and M. Pauly. Axiomatising Nash-consistent coalition logic. In JELIA, pages 394-406, 2002.

[9] H. Moulin and B. Peleg. Cores of effectivity functions and implementation theory. Journal of Mathematical Economics, 10(1):115-145, June 1982.

[10] M. Osborne and A. Rubinstein. A course in Game Theory. The MIT Press, 1994.

[11] M. Pauly. Logic for Social Software. ILLC Dissertation Series, 2001.

[12] I. Seylan and W. Jamroga. Description logic for coalitions. In Proceedings of $A A M A S^{\prime}$ '09, pages 425-432, 2009. 